\title{
Basic science: (March 2009)
}

1. Aaltonen K, Amini RM, Heikkila P, Aittomaki K, Tamminen A, Nevanlinna H, Blomqvist C. High cyclin B1 expression is associated with poor survival in breast cancer. $\mathrm{Br} J$ Cancer 2009; 100: 1055-1060.

2. Al-Bazz YO, Underwood JCE, Brown BL, Dobson PRM. Prognostic significance of Akt, phospho-Akt and BAD expression in primary breast cancer. Eur J Cancer 2009; 45: 694-704.

3. Aurisicchio L, Peruzzi D, Conforti A, Dharmapuri S, Biondo A, Giampaoli S, Fridman A, Bagchi A, Winkelmann CT, Gibson R, Kandimalla ER, Agrawal S, Ciliberto G, La Monica N. Treatment of mammary carcinomas in HER-2 transgenic mice through combination of genetic vaccine and an agonist of toll-like receptor 9. Clin Cancer Res 2009; 15: 1575-1584.

4. Bertucci F, Finetti P, Cervera N, Charafe-Jauffret $E$, Buttarelli $M$, Jacquemier $J$, Chaffanet $M$, Maraninchi D, Viens P, Birnbaum D. How different are luminal $A$ and basal breast cancers? Int J Cancer 2009; 124: 1338-1348.

5. Blevins-Primeau AS, Sun D, Chen G, Sharma AK, Gallagher CJ, Amin S, Lazarus P. Functional significance of UDP-glucuronosyltransferase variants in the metabolism of active tamoxifen metabolites. Cancer Res 2009; 69: 1892-1900.

6. Chisarnore MJ, Wilkinson HA, Flores $\mathrm{O}$, Chen JD. Estrogen-related receptor- $\alpha$ antagonist inhibits both estrogen receptor-positive and estrogen receptor-negative breast tumor growth in mouse xenografts. Mol Cancer Ther 2009; 8: 672-681.

7. Creighton $\mathrm{CJ}$, Osborne $\mathrm{CK}$, van de Vijver MJ, Foekens JA, Klijn JG, Horlings HM, Nuyten D, Wang Y, Zhang Y, Chamness GC, Hilsenbeck SG, Lee AV, Schiff R. Molecular profiles of progesterone receptor loss in human breast tumors. Breast Cancer Res Treat 2009; 114: 287-299.

8. Eubank TD, Roberts RD, Khan M, Curry JM, Nuovo GJ, Kuppusamyl P, Marsh CB. Granulocyte macrophage colony-stimulating factor

First published online 14/07/09 BCO/852/2009/JW inhibits breast cancer growth and metastasis by invoking an anti-angiogenic program in tumor-educated macrophages. Cancer Res 2009; 69: 2133-2140.

9. Finkbeiner MR, Astanehe $A$, To K, Fotovati $A$, Davies $A H$, Zhao $Y$, Jiang $H$, Stratford $A L$, Shadeo A, Boccaccio C, Comoglio P, Mertens $P R$, Eirew P, Raouf A, Eaves CJ, Dunn SE. Profiling YB-1 target genes uncovers a new mechanism for MET receptor regulation in normal and malignant human mammary cells. Oncogene 2009; 28: 1421-1431.

10. Frogne T, Benjaminsen RV, Sonne-Hansen K, Sorensen BS, Nexo E, Laenkholm AV, Rasmussen LM, Riese DJ, de Cremoux P, Stenvang J, Lykkesfeldt AE. Activation of ErbB3, EGFR and Erk is essential for growth of human breast cancer cell lines with acquired resistance to fulvestrant. Breast Cancer Res Treat 2009; 114: 263-275.

11. Gobert M, Treilleux I, Bendriss-Vermare N, Bachelot T, Goddard-Leon S, Arfi V, Biota C, Doffin AC, Durand I, Olive D, Perez S, Pasqual N, Faure C, Coquard IR, Puisieux A, Caux C, Blay JY, Menetrier-Caux C. Regulatory T cells recruited through CCL22/CCR4 are selectively activated in lymphoid infiltrates surrounding primary breast tumors and lead to an adverse clinical outcome. Cancer Res 2009; 69: 2000-2009.

12. Gonzalez L, Zambrano A, Lazaro-Trueba I, Lopez E, Gonzalez JJA, Martin-Perez J, Aranda A. Activation of the unliganded estrogen receptor by prolactin in breast cancer cells. Oncogene 2009; 28: 1298-1308.

13. Hirschfeld $M$, zur Hausen $A$, Bettendorf $H$, Jager $M$, Stickeler E. Alternative splicing of Cyr61 is regulated by hypoxia and significantly changed in breast cancer. Cancer Res 2009; 69: 2082-2090.

14. Hollmen M, Maatta JA, Bald L, Sliwkowski MX, Elenius K. Suppression of breast cancer cell growth by a monoclonal antibody targeting cleavable ErbB4 isoforms. Oncogene 2009; 28: 1309-1319. 
15. Horlock C, Stott B, Dyson PJ, Morishita M, Coombes RC, Savage P, Stebbing J. The effects of trastuzumab on the CD4+CD25+ FoxP3+and CD4+IL17A+T-cell axis in patients with breast cancer. $\mathrm{Br} J$ Cancer 2009; 100: 1061-1067.

16. Iorio MV, Casalini P, Piovan C, Di Leva G, Merlo A, Triulzi T, Menard S, Croce CM, Tagliabue E. MicroRNA-205 regulates HER3 in human breast cancer. Cancer Res 2009; 69: 2195-2200.

17. Loddo M, Kingsbury SR, Rashid M, Proctor I, Holt C, Young J, El-Sheikh S, Falzon M, Eward KL, Prevost T, Sainsbury R, Stoeber K, Williams $\mathrm{GH}$. Cell-cycle-phase progression analysis identifies unique phenotypes of major prognostic and predictive significance in breast cancer. Br J Cancer 2009; 100: 959-970.

18. McCabe N, Cerone MA, Ohishi T, Seimiya H, Lord CJ, Ashworth A. Targeting tankyrase 1 as a therapeutic strategy for BRCA-associated cancer. Oncogene 2009; 28: 1465-1470.

19. Narayan M, Wilken JA, Harris LN, Baron AT, Kimbler KD, Maihle NJ. Trastuzumab-induced HER reprogramming in "resistant" breast carcinoma cells. Cancer Res 2009; 69: 2191-2194.

20. Park SA, Na HK, Kim EH, Cha YN, Surh YJ. 4-Hydroxyestradiol induces anchorageindependent growth of human mammary epithelial cells via activation of I $\kappa$ B kinase: potential role of reactive oxygen species. Cancer Res 2009; 69: 2416-2424.

21. Proietti CJ, Rosemblit C, Beguelin W, Rivas MA, Flaque MCD, Charreau EH, Schillaci R, Elizalde PV. Activation of Stat3 by heregulin/ ErbB-2 through the co-option of progesterone receptor signaling drives breast cancer growth. Mol Cell Biol 2009; 29: 1249-1265.

22. Reddy BY, Greco SJ, Patel PS, Trzaska KA, Rameshwar P. RE-1-silencing transcription factor shows tumor-suppressor functions and negatively regulates the oncogenic TAC1 in breast cancer cells. Proc Natl Acad Sci U S A 2009; 106: 4408-4413.

23. Redmond AM, Bane FT, Stafford T, McLlroy M, Dillon MF, Crotty TB, Hill AD, Young LS. Coassociation of estrogen receptor and p160 proteins predicts resistance to endocrine treatment; SRC-1 is an independent predictor of breast cancer recurrence. Clin Cancer Res 2009; 15: 2098-2106.
24. Sorensen RB, Andersen RS, Svane IM, EngellNoerregaard L, Hadrup SR, Balslev E, Andersen $\mathrm{MH}$, Straten PT. CD8 T-cell responses against cyclin B1 in breast cancer patients with tumors overexpressing p53. Clin Cancer Res 2009; 15: 1543-1549.

25. Umemura S, Shirane M, Takekoshi S, Kusakabe $\mathrm{T}$, Itoh J, Egashira N, Tokuda $\mathrm{Y}$, Mori K, Osamura YR. Overexpression of E2F-5 correlates with a pathological basal phenotype and a worse clinical outcome. Br J Cancer 2009; 100: 764-771.

26. van Agthoven $T$, Veldscholte $\mathrm{J}$, Smid $\mathrm{M}$, van Agthoven TLA, Vreede L, Broertjes M, de Vries I, de Jong D, Sarwari R, Dorssers LCJ. Functional identification of genes causing estrogen independence of human breast cancer cells. Breast Cancer Res Treat 2009; 114: 23-30.

27. Wu XL, Hawse JR, Subramaniam M, Goetz MP, Ingle JN, Spelsberg TC. The tamoxifen metabolite, endoxifen, is a potent antiestrogen that targets estrogen receptor $\alpha$ for degradation in breast cancer cells. Cancer Res 2009; 69: 1722-1727.

28. Xian W, Pappas L, Pandya D, Selfors LM, Derksen PW, de Bruin M, Gray NS, Jonkers J, Rosen JM, Brugge JS. Fibroblast growth factor receptor 1-transformed mammary epithelial cells are dependent on RSK activity for growth and survival. Cancer Res 2009; 69: 2244-2251.

29. Yamnik RL, Digilova A, Davis DC, Brodt ZN, Murphy CJ, Holz MK. S6 Kinase 1 regulates estrogen receptor $\alpha$ in control of breast cancer cell proliferation. J Biol Chem 2009; 284: 6361-6369.

30. Zheng W, Long JR, Gao YT, Li C, Zheng Y, Xiang YB, Wen WQ, Levy S, Deming SL, Haines JL, Gu K, Fair AM, Cai QY, Lu W, Shu XO. Genome-wide association study identifies a new breast cancer susceptibility locus at 6q25.1. Nat Genet 2009; 41: 324-328.

31. Zhou Q, Geahlen RL. The protein-tyrosine kinase Syk interacts with TRAF-interacting protein TRIP in breast epithelial cells. Oncogene 2009; 28: 1348-1356.

Prepared by

$R$ Sutherland

Cancer Research Program

Garvan Institute of Medical Research Darlinghurst, NSW, Australia 